\title{
Responses of Lipids and Lipoproteins Following Acute and Training Resistance Exercise in Obese Postmenopausal Women
}

Ronique N. Pleasant ${ }^{1}$, Melinda Villarreal1, Joshua S. Wooten ${ }^{1}$, Robert M. Hein², Robert D. Menzies², Melody D. Phillips ${ }^{3}$

${ }^{1}$ Texas Woman's University, ${ }^{2}$ John Peter Smith Hospital, Forth Worth, ${ }^{3}$ Texas Christian University

Int J Exerc Sci 2(1): S4, 2009. A single aerobic session and aerobic training can favorably modify lipids and lipoproteins in postmenopausal women, but the effects of a single resistance exercise session (RE) and resistance training (RT) remain equivocal. PURPOSE: To determine the acute effects of RE and chronic effects of 12 weeks of RT on lipid and lipoprotein-cholesterol concentrations in obese, postmenopausal women. METHODS: Sedentary, obese, non-smoking, postmenopausal women, not taking HRT, were divided into either an exercise group $(\mathrm{E}, \mathrm{n}=10 ;$ age $=65.7 \pm 1.8 \mathrm{y} ; \mathrm{BMI}=32.6 \pm 3.5$ $\left.\mathrm{kg} / \mathrm{m}^{2}\right)$ or control group $\left(\mathrm{C}, \mathrm{n}=11\right.$; age $\left.=66.1 \pm 3.0 \mathrm{y} ; \mathrm{BMI}=32.9 \pm 4.3 \mathrm{~kg} / \mathrm{m}^{2}\right)$. Fasting (12 hr) blood samples were collected prior to and $24 \mathrm{hr}$ after the first (BT) and last (AT) exercise session, and at the same time points for C. E performed ten upper and lower body resistance exercises ( 3 sets, 8 rep/set, 80\% 1-RM) 3 times per week for 12 weeks; while $C$ attended education classes twice per week for 12 weeks. Serum was assayed for total cholesterol, triglycerides, LDL-C, HDL-C, HDL2-C, HDL $3-C$ concentrations. A 2 × 2 $x 2$ (group $x$ training period $x$ time) MANOVA was to determine changes in lipid and lipoprotein variables. A $2 \times 2$ (group $x$ time) repeated measures ANOVA was used to assess body composition. RESULTS: The MANOVA revealed no significant changes in serum lipids or lipoproteins following RE or RT. No changes in body composition were observed post-training (P>0.05). Variable Pre-BT $24 \mathrm{hr}$ BT Pre-AT $24 \mathrm{hr}$ AT TC (mg/dl) C E $189 \pm 28217 \pm 55205 \pm 40212 \pm 49201 \pm 48207 \pm 105206 \pm 42195 \pm 106$ Tg (mg/dl) C E $107 \pm 42114 \pm 4096 \pm 49103 \pm 25116 \pm 49129 \pm 92112 \pm 45102 \pm 48$ LDL-C (mg/dl) C E $112 \pm 26140 \pm 51129 \pm 37137 \pm 41118 \pm 40127 \pm 89124 \pm 41120 \pm 88$ HDL-C $(\mathrm{mg} / \mathrm{dl})$ C E $55 \pm 1655 \pm 1257 \pm 1455 \pm 1660 \pm 1353 \pm 1560 \pm 1654 \pm 17$ HDL2-C $(\mathrm{mg} / \mathrm{dl}) \mathrm{C}$ E $36 \pm 1236 \pm 1035 \pm 1034 \pm 1335 \pm 1233 \pm 1433 \pm 1635 \pm 13$ HDL3-C (mg/dl) C E $19 \pm 919 \pm 521 \pm 721 \pm 625 \pm 620 \pm 526 \pm 620 \pm 7$ CONCLUSION: These data suggest that a single RE session and a 12-week RT program have no effect on lipids and lipoproteins. Compared to the effects of aerobic training, resistance exercise related changes in body composition may be necessary to modify lipids and lipoproteins in obese postmenopausal women.

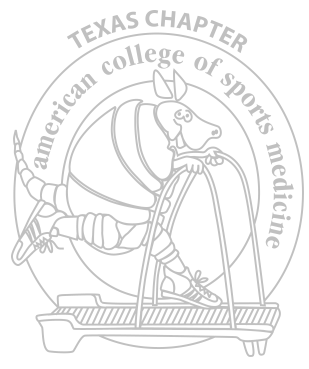

S4 http://www.intjexersci.com 